\title{
Complete humanization of the mouse immunoglobulin loci enables efficient therapeutic antibody discovery
}

\author{
E-Chiang Lee ${ }^{1}$, Qi Liang ${ }^{1}$, Hanif Ali ${ }^{1}$, Luke Bayliss ${ }^{1}$, Alastair Beasley ${ }^{1}$, Tara Bloomfield-Gerdes ${ }^{1}$, Laura Bonoli ${ }^{1}$, \\ Richard Brown ${ }^{1}$, Jamie Campbell ${ }^{1}$, Adam Carpenter ${ }^{1}$, Sara Chalk ${ }^{1}$, Alison Davis ${ }^{1}$, Nick England ${ }^{1}$, \\ Alla Fane-Dremucheva ${ }^{1}$, Bettina Franz ${ }^{1}$, Volker Germaschewski ${ }^{1}$, Helen Holmes ${ }^{1}$, Steve Holmes ${ }^{1}$, Ian Kirby ${ }^{1}$, \\ Miha Kosmac ${ }^{1}$, Anais Legent ${ }^{1}$, Hui Lui ${ }^{1}$, Anais Manin ${ }^{1}$, Siobhan O'Leary ${ }^{1}$, Jemima Paterson ${ }^{1}$, Rocco Sciarrillo ${ }^{1}$, \\ Anneliese Speak ${ }^{2}$, Dominik Spensberger ${ }^{1}$, Laura Tuffery ${ }^{1}$, Nikole Waddell ${ }^{1}$, Wei Wang ${ }^{2}$, Sophie Wells ${ }^{1}$, \\ Vivian Wong ${ }^{1}$, Andrew Wood ${ }^{1}$, Michael J Owen ${ }^{1}$, Glenn A Friedrich ${ }^{1} \&$ Allan Bradley ${ }^{1,2}$
}

\begin{abstract}
If immunized with an antigen of interest, transgenic mice with large portions of unrearranged human immunoglobulin loci can produce fully human antigen-specific antibodies; several such antibodies are in clinical use. However, technical limitations inherent to conventional transgenic technology and sequence divergence between the human and mouse immunoglobulin constant regions limit the utility of these mice. Here, using repetitive cycles of genome engineering in embryonic stem cells, we have inserted the entire human immunoglobulin variable-gene repertoire $(2.7 \mathrm{Mb})$ into the mouse genome, leaving the mouse constant regions intact. These transgenic mice are viable and fertile, with an immune system resembling that of wild-type mice. Antigen immunization results in production of high-affinity antibodies with long human-like complementarity-determining region $3(\mathrm{CDR} 3 \mathrm{H})$, broad epitope coverage and strong signatures of somatic hypermutation. These mice provide a robust system for the discovery of therapeutic human monoclonal antibodies; as a surrogate readout of the human antibody response, they may also aid vaccine design efforts.
\end{abstract}

Antibodies constitute the fastest-growing and most advanced class of pharmaceuticals today ${ }^{1}$. In drug development, antibodies are often preferred over small molecules since they are natural products with exquisite specificity and potency, have a known volume of distribution in the body and generally have superior safety profiles. The first approved therapeutic monoclonal antibodies (mAbs) were derived by humanization of rodent antibodies. More recently, fully human antibodies have been generated using phage libraries and transgenic animals. However, antibodies discovered using phage libraries show limited diversity and non-native pairing of immunoglobulin heavy and light chains; moreover, improving their affinity requires iterative, time-consuming, in vitro methods, which take place outside the natural controls on a mammalian B cell. These antibodies often have suboptimal biophysical attributes, leading to difficulties in manufacture and in poor pharmacokinetics. They can also be immunogenic in patients, leading to attenuation of their efficacy over time.

One attractive approach for generating fully human antibodies is to use transgenic animals engineered to express a human antibody repertoire. This approach allows highly evolved in vivo mechanisms, such as hypermutation in germinal centers, to be exploited to generate high-affinity antibodies with optimal biophysical properties ${ }^{2-4}$. In fact, seven human antibodies approved for clinical use by the US Food and Drug Administration were discovered using transgenic mice first developed over 20 years ago ${ }^{5}$. These mice contain randomly integrated human immunoglobulin heavy-chain $(I G H)$ and kappa light-chain
(IGK) transgenes, as well as targeted loss-of-function alleles of the endogenous mouse Igh and Igk loci ${ }^{6}$. The technology used to establish these first-generation mice had several inherent limitations, including position effects due to the random integration of the transgene and upper limits on the length of the DNA that could be introduced into the mouse genome by means of zygote injection. As a result these mice had incomplete human antibody repertoires. In addition, these first-generation transgenes included both human variable and constant regions. Although these mice would be expected to produce fully human antibodies, there are sequence differences between human and mouse IgM constant regions ${ }^{7}$ and signaling proteins Ig $\alpha$ and $\operatorname{Ig} \beta$. As a result, during the stage when an antibody functions as a B-cell receptor (BCR), the interaction between the human constant region and the mouse signaling proteins $\operatorname{Ig} \alpha$ and $\operatorname{Ig} \beta$ may not be optimal, and reduced signaling could limit antibody class switching and affinity maturation, as well as B-cell differentiation into mature antibody-secreting plasma cells ${ }^{8-10}$.

Here we describe transgenic mice designed to overcome these limitations. We inserted the full complement of variable genes from all three human immunoglobulin loci into precise locations in the corresponding loci of mouse embryonic stem (ES) cells using a technology we developed called sequential recombinase-mediated cassette exchange (S-RMCE). We silenced the endogenous mouse variable genes using a large chromosomal inversion. In these mice, aside from $5.4 \mathrm{Mb}$ of human sequence, no non-mouse DNA remains and none

${ }^{1}$ Kymab Ltd., Babraham Research Campus, Cambridge, UK. ${ }^{2}$ Wellcome Trust Sanger Institute, Hinxton, Cambridge, UK. Correspondence should be addressed to A. Bradley (abradley@kymab.com). 
of the mouse immunoglobulin genes are deleted. Mice carrying these engineered chromosomes are viable and fertile and have a normal immune system. They respond robustly to antigen challenge and produce high-affinity antibodies with human-like CDRH3 lengths, which exhibit broad epitope coverage. The mice produce chimeric antibodies with human variable domains and mouse constant domains. For the production of therapeutic antibodies for the clinic, the mouse constant region can be replaced by a human constant region of choice without loss of affinity or potency. These new mouse strains provide a robust and versatile platform for therapeutic antibody discovery.

\section{RESULTS}

\section{Transgene design}

We inserted the full complement of human variable genes into their respective mouse loci (i) to capture the full diversity encoded by the human immunoglobulin loci and (ii) to achieve physiological expression of the BCR during B-cell ontogeny, which would enable efficient antibody hypermutation and affinity maturation. For the heavy-chain and kappa light-chain loci, we selected an insertion point upstream of the respective mouse constant regions. Variable-diversityjoining $(\mathrm{V}(\mathrm{D}) \mathrm{J})$ recombination would generate chimeric antibodies with human variable and mouse constant regions. BCRs with the mouse constant region should support optimal cell signaling into mouse B cells. Because the lambda light-chain locus has diverged extensively between mouse and humans, we elected to insert the full human locus including the lambda constant regions into the mouse genome immediately downstream of the mouse lambda coding region, leaving the mouse lambda locus unmodified.

After insertion of the human variable regions, the endogenous mouse variable regions remain in the genome and could therefore be incorporated into antibodies during $\mathrm{V}(\mathrm{D}) \mathrm{J}$ recombination. We inactivated the mouse variable region by a chromosomal inversion as it would be unlikely that mouse variable regions that are distant from and inverted relative to the mouse constant region would be used in $\mathrm{V}(\mathrm{D}) \mathrm{J}$ recombination events. We chose inversion rather than deletion because non-immunoglobulin genes with potentially important physiological functions are interspersed among the variable genes of mice and human. For example, Adam $6 a$ and Adam $6 b$ are found between the most $3^{\prime}$ heavy-chain $\mathrm{V}_{\mathrm{H}}$ region and the $\mathrm{D}_{\mathrm{H}}$ cluster. The products of these genes are metalloproteases expressed on the surface of mature sperm and are thought to contribute to sperm migration from the uterus to the oviduct ${ }^{11}$. In separate experiments, we created null mutations of both Adam $6 a$ alone and a combination of Adam $6 a$ and Adam6b. Males homozygous for either mutation have dramatically reduced fertility; indeed, most such males are completely infertile (data not shown). Inactivating the mouse variable region by inversion rather than deletion retains these genes in the mouse genome.

\section{Insertion of human BACs}

We selected a set of overlapping bacterial artificial chromosome (BAC) clones from the RPCI11 library ${ }^{12}$. A total of seven BACs covered the variable portions of the heavy-chain locus, and five and six BACs covered the kappa and lambda light-chain loci, respectively. We subjected the BACs to molecular analysis to confirm their integrity and sequenced them to confirm the presence, functionality and allelic identity of each V, D and J gene (Supplementary Tables 1 and 2). To reduce the number of ES cell manipulation steps, the last two heavy-chain BACs and the last two lambda light-chain BACs were fused to generate large single BACs. Each BAC was trimmed ${ }^{13}$ to remove overlapping sequence. The $3^{\prime}$ ends of the first IGH and IGK BACs were trimmed to a point in the introns adjacent to the most $3^{\prime}$ variable genes. The $I G H$ and $I G K$ human intron enhancers were not included. However, the human $3^{\prime} \mathrm{E}_{\lambda}$ enhancer in the IGL locus was maintained on the first IGL BAC.

The sizes of the three loci $(917 \mathrm{~kb}, 838 \mathrm{~kb}$ and $932 \mathrm{~kb}$ for IGH, IGK and $I G L$, respectively) and the desire to target the human loci precisely into the mouse genome precluded our constructing the transgenes in a single step. We therefore devised S-RMCE, an iterative insertion method (Fig. 1). Using this technique, we inserted the large human BACs using Cre-lox recombination with positive selection ${ }^{14}$. We removed the selection cassettes from the locus using piggyBac transposase with negative selection ${ }^{15}$.

Because many serial genetic manipulation steps are required to insert multiple BACs into the genome, we selected AB2.1 ES cells because they tolerate multiple, sequential genetic manipulation steps while maintaining the ability to colonize the germline ${ }^{16}$. AB2.1 cells are also HPRT deficient, which enables positive selection with an HPRT minigene in hypoxanthine-aminopterin-thymidine (HAT)containing medium. We used HPRT selection for the primary targeting of each landing pad into the three immunoglobulin loci and for the final chromosome engineering step (Fig. 1).

The heavy-chain and the two light-chain loci were engineered separately starting with AB2.1 cells and only brought together through breeding of the resulting animals. S-RMCE was initiated at a landing-pad construct targeted to a position that would delimit the junction between the human and mouse sequences (Fig. 1). We elected to preserve the linear relationship of the mouse intronic $\mathrm{E}_{\mu}$ enhancers, the heavy-chain switch region and the mouse constant region in order to maintain wild-type gene expression. Thus, landing pads were targeted between the last variable J-gene segment and the intronic enhancers $\mathrm{E}_{\mu}$ and $\mathrm{E}_{\mathrm{\kappa}}$ in the Igh and Igk loci, respectively. The landing pad in the lambda light-chain locus was positioned downstream of the most $3^{\prime} \mathrm{J}$-C cluster.

The first human BACs were introduced into ES cells by electroporation and inserted into the landing pad using Cre-mediated recombination between lox sites. By using a wild-type and a mutant (lox5171) lox site, we inserted the BAC in a defined direction. ES cells with the desired BAC insertion events were selected by culturing in puromycin, as the promoterless puro $\Delta T K$ gene in the incoming vector is juxtaposed with the $P G K$ promoter when inserted correctly, whereas random insertion of the BAC into the ES cell genome would rarely result in puro $\triangle T K$ expression. Puromycin-resistant ES cell clones were screened by PCR for the presence of the correct junctions and the BAC-encoded human variable genes (Supplementary Fig. 1a). The targeting efficiency and sizes of the modified BACs are provided in Supplementary Table 3.

The incoming BAC and the landing pad each contain an inverted terminal repeat from the piggyBac transposon (Fig. 1a). At the $3^{\prime}$ end of the insertion, the piggyBac repeats generate a functional transposon that can be removed precisely by transient expression of piggyBac transposase. The PGK-puro $\Delta T K$ selection marker and other non-mouse, non-human sequence is removed with piggyBac transposition. Clones with correct excision (and the absence of a subsequent reinsertion) were resistant to the thymidine analog 5-iodo-1( 2 -deoxy-2'-fluoro- $\beta$-D-arabinofuranosyl) uracil (FIAU) as a result of the loss of the thymidine kinase activity of the puro $\Delta T K$ gene. The $5^{\prime}$ end of the BAC insertion was designed so that correct insertion generates a new landing pad with a loxP and a lox2272 site. This entire process of insertion, selection and piggyBac excision can be repeated with a subsequent BAC engineered with the appropriate lox sites, piggyBac elements and selection markers. Using this technique we inserted 2.7 $\mathrm{Mb}$ of human DNA into the mouse genome distributed among three different loci. 
Figure 1 Engineering of the mouse Igh locus. Human sequences are shown in red, mouse sequences are shown in black, and other exogenous sequences are shown in blue or green. (a) Schematic of S-RMCE. First, a vector encoding two incompatible target lox sites (one wild-type loxP and one mutant lox5171) is inserted into the Igh locus in mouse ES cells; this provides a 'landing pad' (blue-outlined box). Next, the first BAC (BAC1) encoding a portion of the human IGH variable region is inserted directionally at these lox sites in the landing pad by Cre-mediated recombination. Correct integrations juxtapose the $P G K$ promoter (denoted PGK) functionally next to the purosTK gene, enabling puromycin expression and positive selection for puromycin-resistant clones. In puromycin-resistant clones, the $3^{\prime}$ side of the insertion now has an intact piggyBac transposon (PB) with both $5^{\prime}$ and $3^{\prime}$ inverted terminal repeats (shaded gray). Expression of piggyBac transposase results in excision of this element. Clones which have excised the PB transposon lose the puro $\Delta T K$ gene and therefore thymidine kinase expression, facilitating negative selection in medium containing FIAU. The $5^{\prime}$ end of the insertion recreates a new landing pad with a wild-type loxP and a mutant lox2272 site (shaded blue), allowing integration of additional BACs in an iterative process. Six junctionspecific primers, P1-P6, are used to confirm the integrity of the insertions. (b) Schematic of a mouse V-region-inactivating inversion. After insertion of the last human BAC in each locus, an inversion end point is targeted onto the same chromosome several megabases distant (greenoutlined box). This provides a loxP site that is inverted relative to the loxP in the landing pad such that expression of $\mathrm{Cre}$ inverts the intervening region. The inversion forms an intact HPRT minigene, enabling positive selection for clones with these events in medium containing sodium hypoxanthine, aminopterin and thymidine (HAT). The inversion also generates two intact PB transposons. These are simultaneously excised by piggyBac transposase expression and negatively selected in FIAU. All exogenous sequences (with the exception of the human sequences intentionally inserted into the mouse genome) are removed from the locus. F1 and F2 refer to the two most distal cytogenetic bands on mouse chromosome 12 .

\section{Inversion of endogenous Ig loci}

We analyzed the species origin of $\mathrm{V}, \mathrm{D}$ and J segment usage in mRNA transcripts of antibody genes in mice with human BACs inserted via S-RMCE. No mouse D or J segments were found in heavy-chain or kappa light-chain transcripts. However, we detected both human and mouse V-segment sequences in $I G H$ and $I G K$ transcripts. The frequency of mouse $\mathrm{V}$-segment usage diminished as more BACs were inserted (Fig. 2a). Nevertheless, to prevent mouse V-segments from being used to generate antibodies in the transgenic mice, we created inversions in the heavy-chain and kappa light-chain loci. The inversions moved the entire mouse variable gene clusters several megabases away on the same chromosome and inverted them relative to the mouse constant regions.

To engineer this inversion at the heavy-chain locus (Fig. 1b), we targeted vectors to positions $6.25 \mathrm{Mb}$ distal to the inserted human $I G H$ locus and $26.3 \mathrm{Mb}$ proximal to the inserted human IGK locus (proximal and distal are relative to the centromere). The targeted positions were chosen to avoid disrupting any functional genes near the inversion break points. Transient expression of Cre generated the inversion and united two halves of an HPRT minigene, enabling selection in HAT (Supplementary Table 4) ${ }^{16}$. HAT-resistant clones were analyzed by PCR to confirm that they created the expected junctions. The endpoint cassettes were designed so that the rearrangement reconstituted two functional piggyBac transposons encompassing all of the cassette sequence including the selection markers. piggyBac transposase expression and selection for the loss of HPRT using 6-thioguanine were then used to delete the transposons, eliminating all cassette DNA and leaving seamless junctions at either end of the inversion. The final structures of the transgenic loci are depicted in Supplementary Figure 2.

\section{Generation of transgenic mice}

ES cell lines resulting from each S-RMCE step were used to generate transgenic mice by standard procedures. All variable elements and each insertion and inversion junction were checked in ES cells and confirmed in the genome of resulting mice (Supplementary Fig. 1b). Animals homozygous for each allele were obtained at the expected Mendelian ratios and were fertile.

Mice with transgenic heavy- and light-chain alleles were crossed to establish two true-breeding strains. HK mice are homozygous for both humanized heavy- and kappa light-chain alleles and an unmodified mouse lambda light-chain locus. HL mice are homozygous for 
Figure 2 Human and mouse variable region usage in transgenic mice. Shown is V-region usage in antibody transcripts as determined by cloning and sequencing 5' RACE products synthesized from RNA isolated from spleens of the indicated transgenic mice. The reverse transcription reactions were primed from $\mathrm{C} \mu$-specific or $\mathrm{C} \kappa$-specific primers. All the heavy-chain transcripts used the human $D_{H}$ and $J_{H}$ segments, and all the kappa light-chain transcripts used human $J_{K}$. (a) V-region usage in mice with 1-3 IGH BACs or IGK BACs, without any inversions of endogenous immunoglobulin. (b) V-region usage in $\mathrm{HK}$ and $\mathrm{HL}$ mice with inversions of the endogenous $\operatorname{lgh}(\mathrm{HK}$ and $\mathrm{HL}$ ) and $\operatorname{lgk}$ loci $(\mathrm{HK})$. Sample size $(n)$ is provided for each data point.

both humanized heavy- and lambda light-chain alleles and have a homozygous knockout of the kappa locus. The genetic background of these fertile, true-breeding lines is a randomized mixture of 129S7 and $\mathrm{C} 57 \mathrm{BL} / 6 \mathrm{~J}$ strains; however, the linkage of the transgenes to the constant regions of the heavy- and kappa light-chain loci mean that the constant regions are always from the 129S7 strain. Both background strains and $\mathrm{HK}$ and HL lines are homozygous for the $\mathrm{H}-2^{\mathrm{b}} \mathrm{MHC}$ haplotype, but they will differ at other minor histocompatibility antigens.

\section{Human and mouse V-gene usage}

The degree to which the inversions reduced the use of mouse variable $(\mathrm{V})$ genes was determined by analysis of transcripts from splenocytes of naive $\mathrm{HK}$ and HL mice. Antibody-encoding transcripts were reverse transcribed using $\mathrm{C} \mu$ and $\mathrm{C} \kappa$ constant region-specific primers and amplified with $5^{\prime}$ rapid amplification of cDNA ends $\left(5^{\prime}\right.$ RACE) primers. Sequencing showed that over $99.7 \%$ of heavy-chain transcripts in both HK and HL mice used human V-gene segments. About $98 \%$ of kappa light-chain transcripts in HK mice used human $\mathrm{V}$ genes and $87 \%$ of lambda light-chain transcripts used human $\mathrm{V}$ genes in HL mice. In HL mice the mouse kappa locus was fully inactivated by insertion of a neo ${ }^{\mathrm{R}}$ cassette without deletion of any endogenous kappa variable region DNA (Supplementary Fig. 3). The insertion of the human lambda BACs into the lambda locus, however, does not disrupt the three endogenous mouse $\mathrm{V}_{\lambda}$ segments. In $12 \%$ of transcripts with the human lambda constant region, one
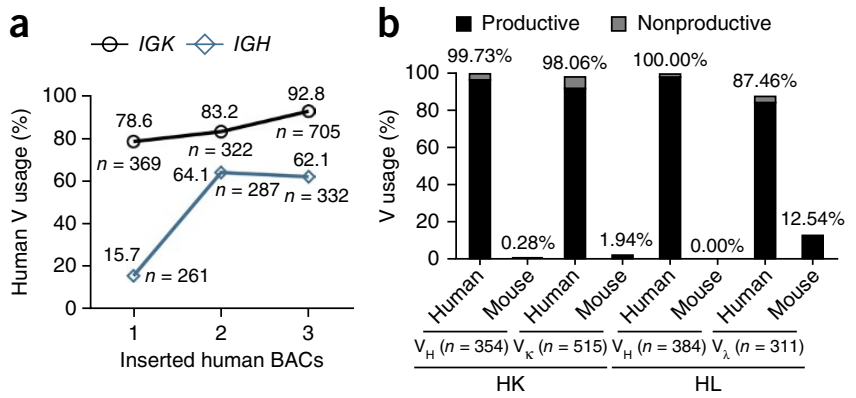

of these three mouse variable segments is found along with a human $\mathrm{J}_{\lambda}$ segment (Fig. $\mathbf{2 b}$ ).

All of the inserted human heavy- and light-chain variable regions were used in HK and HL mice. The relative frequency of usage of each element varied considerably, as expected (Supplementary Figs. 4 and 5). In $\mathrm{HK}$ mice, for example, the human $\mathrm{V}_{\mathrm{H}} 6-1$ variable region is found in $16.5 \%$ of transcripts and $\mathrm{V}_{\mathrm{H}} 1-2$ in $0.9 \%$. The pattern of $\mathrm{V}_{\mathrm{H}}$ gene use differs slightly between $\mathrm{HK}$ and $\mathrm{HL}$ mice (Supplementary Fig. 4a). This is likely a consequence of the different biophysical properties of the kappa and lambda light chains, whose pairing with heavy chains may affect $V_{H}$ usage. HK mice have two human $V_{\kappa}$ genes from the distal inverted $V_{\kappa}$ gene set, which are infrequently used in humans. To correct this ancient evolutionary rearrangement, these genes have been inserted closer to the proximal $\mathrm{V}_{\mathrm{K}}$ genes and reoriented so that they are transcribed in the correct direction. As a result the frequency with which these distal $V_{K}$ genes are used in our transgenic mice is elevated relative to that in humans (Supplementary Fig. 5a).

\section{B-cell development and maturation in transgenic mice}

We used a variety of measures to compare the immune system in these transgenic mice to that of wild-type, age-matched mice of similar genetic background. The spleen, bone marrow and lymph nodes were normal in appearance, size and absolute cell numbers (Supplementary Fig. 6). When analyzing IgM and IgD expression on $\mathrm{B} 220^{+}$cells in the spleen and lymph nodes, we detected no
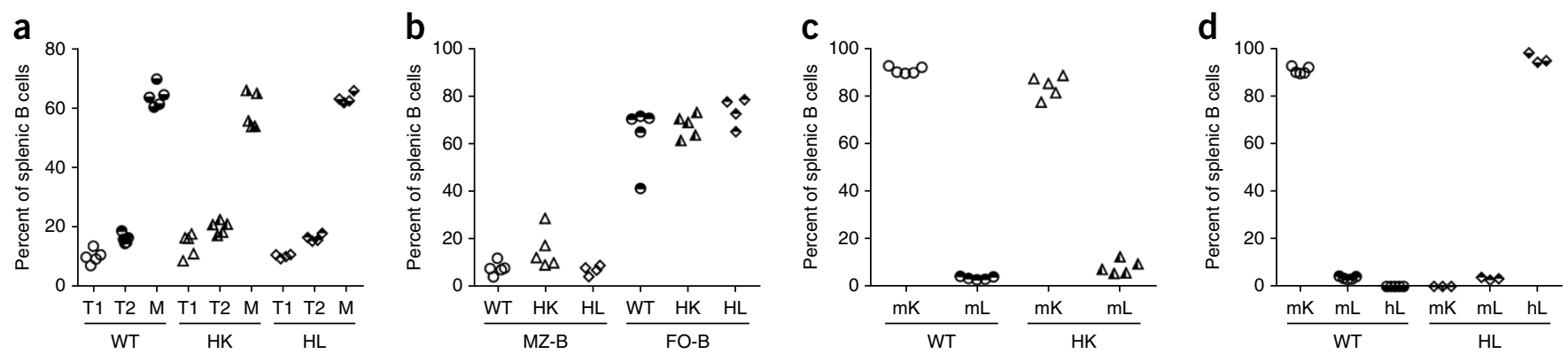

Figure 3 Normal B-cell maturation and antibody production in HK and HL mice. (a) Transitional type 1 (T1), transitional type 2 (T2) and mature (M) B-cell populations as quantified by flow cytometric analysis of expression of IgM and IgD on the surface of $\mathrm{B}_{220^{+}}$splenocytes. (b) Marginal-zone (MZ) and follicular (FO) B cells as quantified by flow cytometric analysis of expression of CD21 and $\mathrm{CD} 23$ on the surface of $\mathrm{B} 22 \mathrm{O}^{+}$splenocytes. (c,d) Proportion of splenic B cells in $\mathrm{HK}$ (c) and HL (d) mice expressing different light chains ( $\mathrm{mK}$, mouse kappa chain; $\mathrm{mL}$, mouse lambda chain; hL, human lambda chain) compared to wild-type (WT) controls as determined by staining with antibodies specific for each light-chain constant region. (e) Serum concentration of different classes of antibody (IgM, open shapes, and IgG1, IgG2a and IgG2b combined,
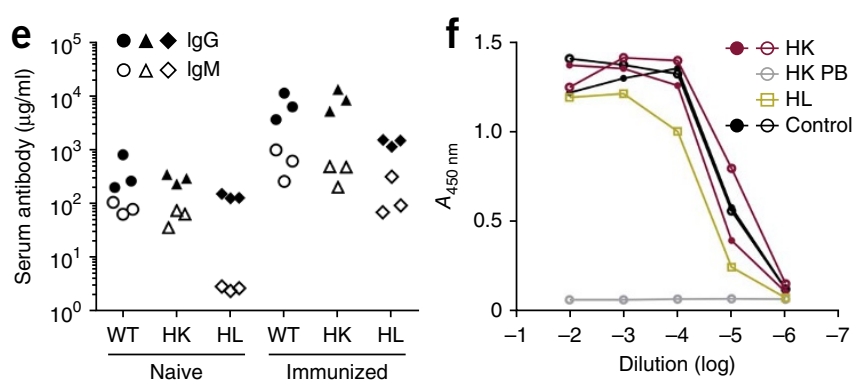
closed shapes) in naive and CD4OL-immunized $\mathrm{HK}$ and $\mathrm{HL}$ mice as compared to a WT control. (f) Titers of CD4OL-specific polyclonal antibodies in the serum of immunized mice as determined by ELISA. PB, pre-bleed or naive serum sample; controls are non-transgenic animals of the same mixed strain background. 
a

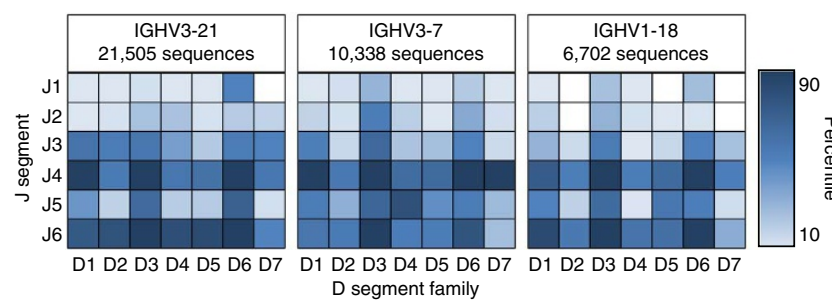

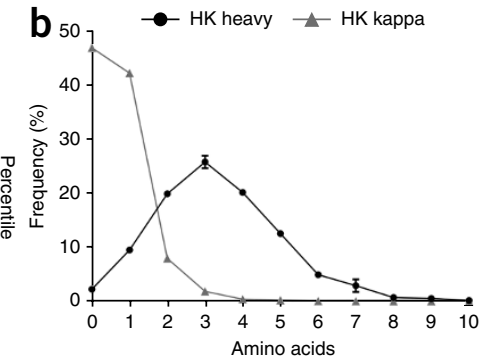

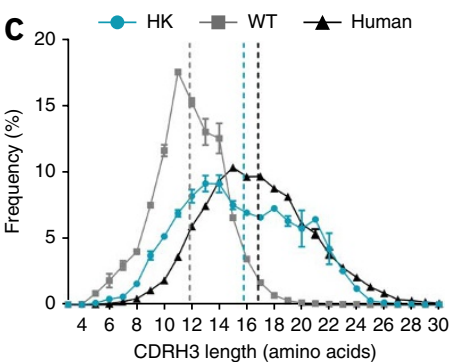

Figure 4 Sequence analysis of antibody transcripts in HK mice. (a) Heat map depicting the frequency of particular V-D-J combinations among transcripts encoding three frequently used human $\mathrm{V}_{\mathrm{H}}$ segments. (b) Numbers of amino acids added as a result of P-and N-nucleotide additions at the junctions of $\mathrm{V}$-D and D-J segments in heavy-chain transcripts (black) and V-J segments in kappa light-chain transcripts (gray). Sequences from two HK mice were analyzed. (c) CDRH3 lengths determined from sequences of antibody transcripts from the spleens of two HK (blue) and two wild-type (WT) control (gray) mice and two human peripheral blood samples (black). The mean for each set is depicted by a dashed vertical line. Error bars delimit the range of values for the two samples analyzed.

significant differences in the proportion of transitional type 1 (T1), transitional type 2 (T2) and mature (M) B cells in HK and HL mice compared to wild-type controls (Fig. 3a and Supplementary Fig. 7a). The proportion of splenic marginal zone and follicular B cells was also normal (as determined by CD21 and CD23 staining; Fig. 3 b and Supplementary Fig. 7 b).

The endogenous Igl locus is not inactivated in HK or HL mice, so it was possible that if there was a deficiency in the transgenic human kappa locus, this might be reflected by a relative increase in mouse lambda light-chain use. HK mice did have slightly more B cells producing lambda light-chain antibodies (5-10\% compared to $2-5 \%$ in wild-type; Fig. 3c and Supplementary Fig. 7c), whereas HL mice did not (Fig. 3d). Over $90 \%$ of the B cells in the HL mice expressed lambda light chain with a human lambda constant region. Further analysis of the B-cell compartments in HK mice showed normal development of pro- $\mathrm{B}$, pre-B and immature $\mathrm{B}$ cells in the bone marrow (Supplementary Fig. 8). T cell subpopulations, natural killer (NK) and NKT cells, neutrophils, eosinophils and monocytes were present in similar proportions in peripheral blood of HK, HL and wild-type mice (Supplementary Table 5).

\section{Primary antibody repertoire}

The CDRH3 region of the heavy chain of an antibody is encoded by the combination of the V, D and J segments. Considering all V, D and $\mathrm{J}$ segments, the potential combinatorial diversity of the heavy-chain locus in HK and HL mice is $>3,000$; the light-chain loci exceed 200 possible configurations. For the most frequently used variable genes, we observed all possible combinations with $\mathrm{D}$ and J genes (Fig. 4a). However, even at over 30,000 sequences, the sequence set was not extensive enough to observe all possible combinations of less frequently used variable regions.

Combinatorial diversity aside, the largest contributor to the diversity of the CDRH3 region is addition of non-templated $(\mathrm{N})$ and palindromic $(\mathrm{P})$ nucleotides at junctions between segments; nucleotides can also be deleted. We compared the sequence of each CDRH3 to the germline sequence to determine the extent of these processes in $\mathrm{HK}$ and HL mice. More than half of kappa light chains had one or two nongermline amino acids, increasing diversity 400 -fold beyond the combinatorial diversity. As expected, the heavy chain was much more diverse: $69 \%$ of heavy chains had more than three amino acids added, and 20\% had 5-7 added (Fig. 4b). On the basis of the actual use of non-germline amino acids in the heavy chain, we calculated the Shannon entropy $\left(H^{\prime}=-\sum_{i=1}^{N} p_{i} \log _{2}\left(p_{i}\right)\right)$ of 3.90 bits for an effective first-order diversity of 14.9 for the 20 possible amino acids. Using this value, for the set of heavy chains with three additional amino acids (27\% of the total set), there are 3,310 possible combinations, and for those with five additional amino acids (13\%), there are 736,000 possible combinations. Using a weighted calculation encompassing approximately $80 \%$ of the sequence set (2-5 added residues), we determined that these non-germline amino acids increase heavy-chain diversity by more than 132,000 -fold beyond the combinatorial diversity.

The length of the CDRH3 is an important attribute of an antibody, as longer CDRH3s enable antibodies to contact embedded epitopes within single proteins or multisubunit complexes like channels. Human antibodies have a mean CDRH3 length of about 17 amino acids, with a broad distribution between 9 and 24 amino acids. Antibodies with long CDR3Hs have been reported to be beneficial; an example is an antibody with a 24 -residue $\mathrm{CDRH} 3$ that neutralizes many strains of influenza by inserting into the hemagglutinin receptor-binding pocket ${ }^{17}$. We compared the CDRH3s from the HK and HL mice with those from two wild-type mice and two humans (Fig. 4c, and data not shown). Antibodies from HK and HL mice had CDRH3s with a mean length of 16 amino acids, which is about 4 amino acids longer than the average CDRH3s from wild-type mice and 1 amino acid shorter than those of humans. In addition, whereas wild-type mice showed a relatively tight distribution of CDRH3 lengths, HK and HL mice, like humans, had a broad range, with many antibodies having CDRH3s exceeding 24 amino acids.

\section{Robust immune response}

Several HK and HL mice and intermediate prototypes were immunized with a variety of antigens. In all cases $(n=81$ mice), we observed a response as measured by titers of antigen-specific antibodies in serum. In non-immunized HK, HL and wild-type mice, the serum concentrations of total IgM and IgG were $2.4-104.8 \mu \mathrm{g} / \mathrm{ml}$ and 124.5-796.7 $\mu \mathrm{g} / \mathrm{ml}$, respectively. After immunization, IgM and IgG levels in all mice increased to $68.7-975.8 \mu \mathrm{g} / \mathrm{ml}$ and $1.1-12.9 \mathrm{mg} / \mathrm{ml}$, respectively (Fig. 3e,f). The high IgG concentrations and antigenspecific antibody titers indicate that $\mathrm{HK}$ and HL mice underwent class switching.

For example, mice were immunized with human CD40L (CD154) protein in PBS via intraperitoneal injection using the Sigma adjuvant system. We detected CD40L-specific IgGs in the serum of all mice at a dilution of at least 1 in $10^{5}$ (Fig. 3f). Although we noted considerable variability in serum immunoglobulin concentrations in all mice, naive HL mice consistently had lower IgM levels; nevertheless, IgM concentrations increase substantially after immunization. When HL mice are used for mAb discovery, they respond robustly to 
Figure 5 Analysis of V-region sequences of ovalbumin-specific mAbs isolated from prototype mice with two or three heavy-chain BACs and an inversion. (a) Histogram shows the number of amino acid changes at each position in the heavy-chain variable region, as compared to the germline sequence, for 74 individual mAbs. Amino acid numbering is based on the Immunogenetics (IMGT) scheme ${ }^{31}$. (b) Alignment of $\mathrm{V}_{H}$ amino acid sequences from five anti-ovalbumin mAbs of the same $V_{H}$ class carrying near-identical $\mathrm{CDRH} 3 \mathrm{~s}$. Amino acids that differ from the common germline $V_{H}$ are indicated, and identical residues are denoted by a dash. $K_{D}$ values were determined by surface plasmon resonance (SPR) using purified recombinant antibody and purified ovalbumin ('+pos' indicate that weak binding was observed but $K_{\mathrm{D}}$ values were not determined). (c) Dendrogram showing the sequence relationship and distance (number of amino acid changes in square brackets) from germline for each of these five mAbs. FR1, FR2 and FR3, framework regions; CDR1, CDR2 and CDR3, complementarity-determining regions.

immunogens, provide splenocytes that efficiently make hybridomas and have been used to discover several high-affinity, potent $\mathrm{mAbs}$ (data not shown).

The affinity of antigen-antibody interactions is increased by several orders of magnitude through the process of somatic hypermutation mediated by activation-induced cytosine deaminase (AID). To assess the degree of affinity maturation in antibodies from $\mathrm{HK}$ mice, we isolated mAbs that bound to various antigens, measured their affinity and compared their sequence to germline. Extensive somatic hypermutation was evident in all of the IgGs examined, with the mutations clustering in the classically defined CDRH1, CDRH2 and CDRH3 regions (Fig. 5a). In certain cases, we isolated sets of antibodies with related sequences sharing the same CDRH3. Comparing these sequences to the antigen affinities of these antibodies provides
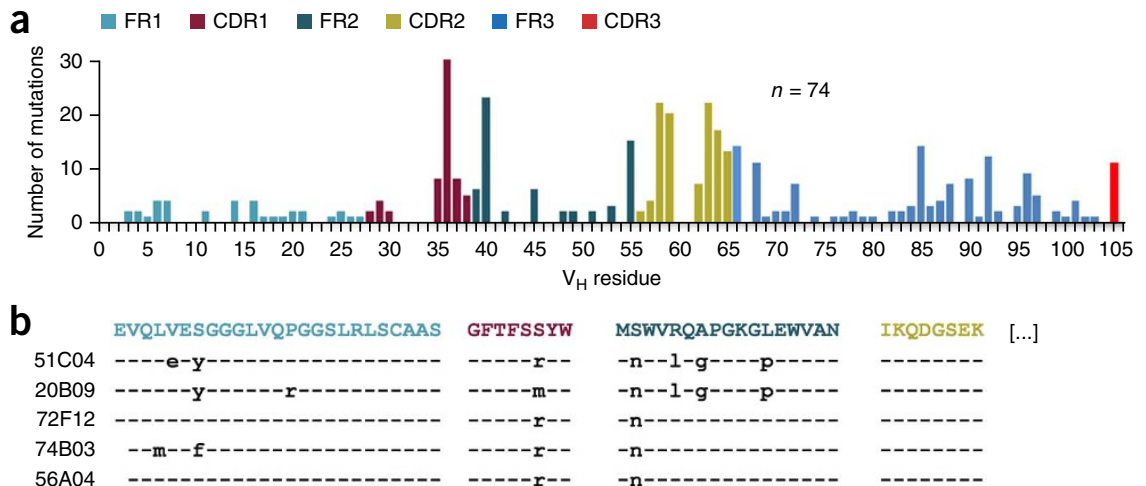

[...] YYVDSVKGRFTISRDNAKNSLYLQMNSLRAEDTAVYYC AR-YSSGW-YYYYYGMDV WGQGTTV --Sf----G---f----- -----S- $\quad<10 \mathrm{pM}$ --Sf----G---f----- ------F $1.0 \mathrm{nM}$ --Sf----G---f----- ---LPRS $19.0 \mathrm{nM}$

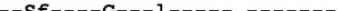
+ pos
+ pos

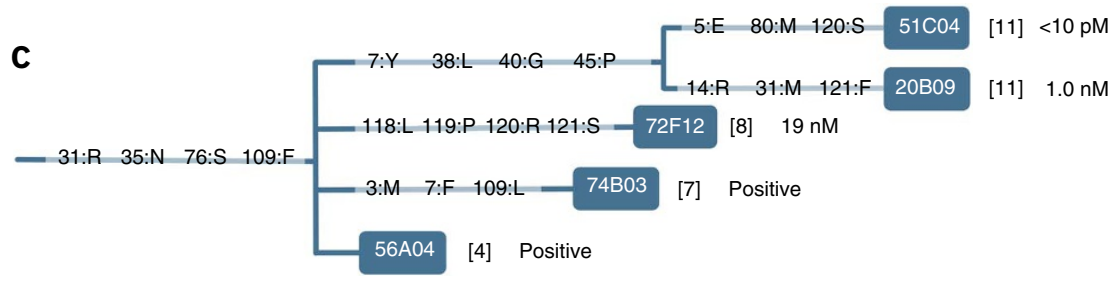

evidence for affinity maturation since it is likely that these antibodies originated from a single early B-cell clone (Fig. $5 \mathbf{b}, \mathbf{c})$.

\section{High-affinity antibodies with broad epitope coverage}

To determine whether HK and HL mice will be useful for discovering potent neutralizing therapeutic mAbs, we immunized them with three antigens: human CD40L, Staphylococcus aureus hemolysin A (Hla) and chicken ovalbumin (OVA); we then isolated panels of mAbs specific for each antigen.

Despite the small scale of these experiments (fewer than ten animals for any antigen), we isolated many antibodies with high affinities,
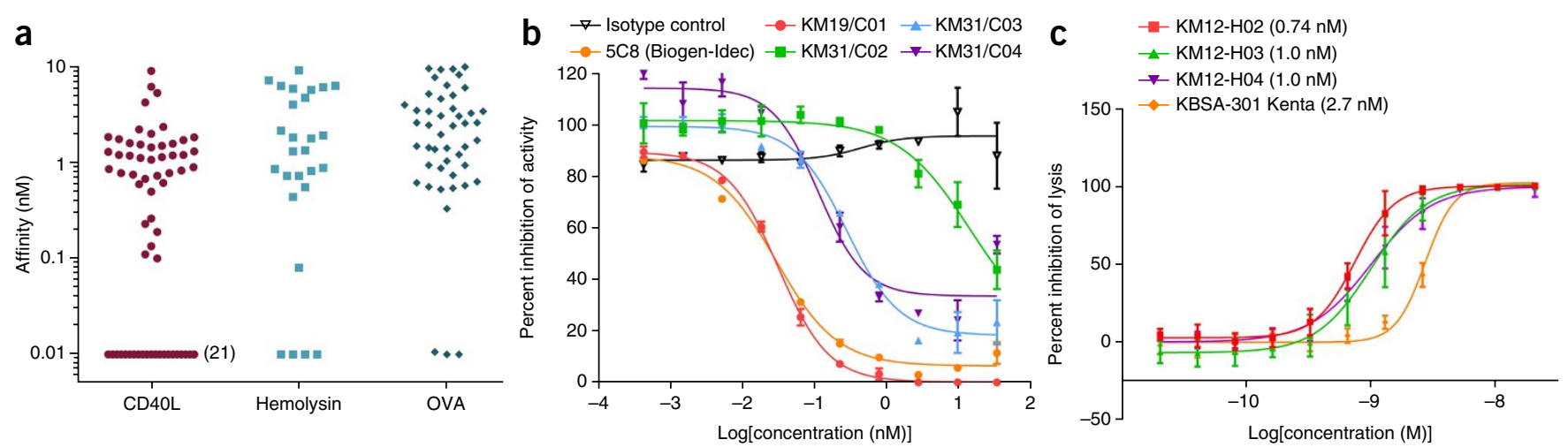

Figure 6 Binding and function of mAbs isolated from HK mice. (a) $K_{D}$ values of mAbs isolated from HK mice immunized with recombinant human CD4OL, recombinant Staphylococcus aureus hemolysin alpha (Hla) or ovalbumin (OVA), determined by SPR. Each symbol represents one mAb. Values shown on the $0.01 \mathrm{nM}$ line (21 in the case of anti-CD40L mAbs) could not be accurately determined because of a limitation of the ProteOn XPR36 in measuring affinities of antibodies with long off-rates. The $K_{D}$ values for CD4OL are apparent and not actual because CD4OL is a trimer. (b) Potency of recombinant anti-CD4OL mAbs isolated from HK mice (KM19/C01, KM31/C03, KM31/C02, KM31/C04), evaluated by neutralization of a CD40Linduced NF-KB-mediated alkaline phosphatase reporter gene assay and compared to the benchmark therapeutic antibody $5 \mathrm{C} 8$. KM19/C01 has an $\mathrm{IC}_{50}$ of $0.031 \mathrm{nM}$ and $5 \mathrm{C} 8,0.029 \mathrm{nM}$. Error bars represent the range of values for duplicate measurements. (c) Potency of recombinant anti-Hla mAbs isolated from $\mathrm{HK}$ mice (KM12-H02, KM12-H03, KM12-H04), evaluated in an assay measuring the blocking of Hla-mediated membrane pore formation and lysis of rabbit RBCs. The benchmark antibody, KBSA-301, originated from human B cells and is currently in clinical trials. Values in parentheses represent the $\mathrm{IC}_{50}$ for each $\mathrm{mAb}$. Error bars depict the s.d. of two repeated experiments in which each sample was analyzed in duplicate. 
in the low nanomolar to low picomolar range (Fig. 6a), verifying the efficiency of affinity maturation in these mice. We used a reportergene assay designed to detect disruption of CD40-CD40L signaling to identify CD40L-neutralizing antibodies and benchmark them against $5 \mathrm{C} 8$, an anti-CD40L mAb developed by Biogen Idec for clinical studies $^{18}$. Several antibodies showed comparable or more potent neutralization of CD40L function (Fig. 6b). Analysis of the epitope specificity of seven of these CD40L-neutralizing antibodies revealed three different epitope 'bins', two of which overlap with the epitope bound by $5 \mathrm{C} 8$ and one of which was unique (data not shown).

Anti-Hla antibodies from an intermediate prototype of the $\mathrm{HK}$ mice (transgenic for 6 human $\mathrm{V}_{\mathrm{H}}$ and $12 \mathrm{~V}_{\mathrm{K}}$ segments) had superior or comparable $\mathrm{EC}_{50}$ values when compared with KBSA-301, an anti-Hla antibody currently in clinical trials (isolated from human B cells by Kenta Biotech with a reported affinity for Hla of $1.4 \mathrm{nM}$ (ref. 19) (Fig. 6c). We reconfigured this $\mathrm{mAb}$ with a mouse constant region, and the reconfigured version had an affinity for Hla of 180 pM. KBSA-301 has undergone extensive affinity maturation, with 11 and 5 amino acid substitutions from the germline sequence in its heavy and light chains, respectively. These mutations may have originated via affinity maturation or may have been engineered into the molecule. AntiHla antibodies isolated from prototype $\mathrm{HK}$ mice had up to eight and four amino acid substitutions in their heavy and light chains, respectively. Anti-Hla antibodies isolated from Velocimmune mice ${ }^{20,21}$ are described in the patent and scientific literature with listed $K_{\mathrm{D}}$ values of between 337 and $601 \mathrm{pM}$. One of these was subjected to affinity maturation by CDR mutagenesis to derive a candidate with higher affinity for clinical development called LC10 (ref. 21). LC10 was derived from a small set of leads all of which had the same lightchain $V_{\kappa}$ and $V_{H}$ regions with 3-5 amino acid differences from the germline sequence.

The HK and HL strains have now been used to isolate lead monoclonal antibodies against a total of eight protein drug targets such as membrane receptors and secreted molecules (data not shown). Many of these campaigns required only a few immunized mice from which hybridomas were used to screen for antibodies with a number of specific criteria. In the case of CD40L and Hla as well as six other drug targets, the antibodies discovered using HK and HL mice have qualities that make them suitable for clinical development.

\section{DISCUSSION}

Transgenes randomly inserted into the genome are influenced by their location, and only rarely will they fully recapitulate normal gene expression $^{22}$. The size limit of conventional transgenes means that substantial portions of the human antibody repertoire are not represented in first-generation human immunoglobulin transgenic mice; this limits the diversity of antibodies these mice could produce. A subsequent generation of transgenic animals carry the entire human heavy-chain locus on a minichromosome ${ }^{23}$. Although these mice offer the potential advantage of a full heavy-chain repertoire, the minichromosome is mitotically and meiotically unstable. Similar instability has been observed for other minichromosomes in mice ${ }^{24}$.

Since diverging from a common ancestor more than 100 million years ago, the immunoglobulin loci have been subject to strong evolutionary selection so that sequences encoding the constant regions of the different human IgG classes are more similar to each other than to any of the mouse IgG constant regions. This divergence may influence the interaction of BCRs containing human constant regions with mouse signaling proteins such as $\operatorname{Ig} \alpha$ and $\operatorname{Ig} \beta$, as well the interactions between antibodies and mouse Fc receptors; this would affect many aspects of B-cell development, function and differentiation ${ }^{25}$.
A solution to this problem is to retain the mouse constant regions while inserting human variable regions into the mouse genome. We are unaware of any other peer-reviewed publications describing mice with such a hybrid immunoglobulin locus.

Recently, a rat strain harboring chimeric transgenes encoding un-rearranged human variable genes upstream of a rat constant region was described ${ }^{26}$. Rats with multiple copies of this transgene have a near-normal B-cell compartment, and the antibodies they produce after immunization display some evidence of hypermutation. However, because classic pronuclear microinjection was used to generate this strain, the rats exhibit some of the limitations of the earlier transgenic mice, namely an incomplete antibody repertoire and multicopy, randomly integrated transgenes. The advantage, if any, of retaining a rat constant region was not resolved in this study.

To capture the full human antibody repertoire and to recapitulate all of the attributes of the complex regulation of the immunoglobulin locus, we introduced the full set of human variable genes into the mouse locus. To construct such a locus we used recombineering technologies to manipulate large human genomic BAC clones in Escherichia coli ${ }^{13,27}$ together with RMCE to insert these large segments of DNA into the ES cell genome ${ }^{28}$. We also exploited the ability of piggyBac transposons to excise themselves cleanly from the genome to remove all foreign nonhuman DNA, enabling us to recycle selection markers ${ }^{15}$. Using this combination of technologies, which we refer to as S-RMCE, we inserted the full repertoire of human immunoglobulin variable genes (2.7 Mb of DNA) into the mouse genome. Up to 15 successive genetic modifications per locus was required; to our knowledge this is the largest number of modifications achieved in an ES cell while still retaining the ability to repopulate the germline.

This efficient technology for inserting large segments of human DNA into homologous segments of the mouse genome may be applied to other complex loci, such as those encoding the T cell receptor, the major histocompatibility complex, the Fc receptors and the complement system. One could therefore, for example, build preclinical models to test the efficacy of active or passive vaccination strategies.

Insertion of the human variable segments does not completely prevent usage of the mouse variable genes retained upstream. Inversions that moved the entire physical expanse of DNA-encompassing all the mouse variable regions (and interstitial non-immunoglobulin genes) - to another location on the same chromosome, millions of base pairs away and in the opposite orientation, were used to silence endogenous mouse heavy-chain and kappa light-chain V-gene usage. The mouse lambda locus is naturally silent and therefore required no further modification. Mice that are homozygous for either or both the heavy-chain and kappa light-chain inversions are phenotypically normal and have wild-type fertility. Non-immunoglobulin genes found among the variable region genes such as Adam6a and Adam6b, whose deletion results in infertility ${ }^{29}$, were unaffected by the inversion.

Here we describe two strains of mice: HK harbors the human heavy-chain and kappa light-chain loci, and HL harbors the human heavy-chain and lambda light-chain loci. B-cell development in naive and immunized HK and HL mice was indistinguishable from that in wild-type mice. Antibody diversity in these mice would therefore not be expected to be limited by an abnormally low B-cell count. Sequence analysis of antibody transcripts from HK and HL mice demonstrated that all introduced human variable elements were used. For $\mathrm{V}_{\mathrm{H}}$ regions that were sufficiently represented, all were found in every possible combination with each $\mathrm{D}_{\mathrm{H}}$ and $\mathrm{J}_{\mathrm{H}}$ segment. Amino acids were added at the junctions of variable regions, yielding antibodies in antigen-naive HK and HL mice with encoded $\mathrm{CDRH} 3 \mathrm{~s}$ that averaged 16 amino acids in length-nearly the average 
length of human $\mathrm{CDRH} 3 \mathrm{~s}$ - an important indicator of the potential utility of these mice as a human antibody-discovery platform. Antibodies with long CDRH3s (up to 24 amino acids in HK mice) can bind deeply embedded epitopes, such as those on heavily glycosylated viral proteins and membrane-embedded antigens such as GPCRs and channels ${ }^{30}$.

Immunized HK and HL mice responded to several different antigens by producing $\mathrm{mAbs}$ that bind to these antigens with high affinity and whose sequence revealed normal signatures of somatic hypermutation and class switching. The isolated antibodies showed broad coverage of epitopes in each antigen, and potent neutralizing antibodies could be readily identified. A comparison between antibodies isolated from HK mice and anti-CD40L and antiHla antibodies developed for clinical applications by other companies demonstrates that antibodies with equivalent or superior properties can be rapidly isolated from HK and HL mice. In addition, unlike some of the benchmark antibodies, antibodies from HK mice do not require time-consuming cycles of iterative engineering to improve their affinity, solubility, expression or other features.

$\mathrm{HK}$ and HL mice thus offer substantial advantages over previously described human immunoglobulin transgenic rodents. We believe that these mouse strains will have broad utility for therapeutic antibody discovery. The human antibodies produced by these mice might also represent an accurate surrogate of the human immune response to candidate vaccine antigens; therefore, analyzing candidate vaccines in these mice may help accelerate and improve vaccine development. To make these mouse strains available to the research community, we have created access routes for commercial and academic entities; details of the academic access program can be found at http://www. kymabaccess.org/.

\section{METHODS}

Methods and any associated references are available in the online version of the paper.

\section{Accession codes. Sequence data: EBI: HG940547-HG940622.}

Note: Any Supplementary Information and Source Data files are available in the online version of the paper.

\section{ACKNOWLEDGMENTS}

We thank A. Sandham, J. Clube and D. Casson for frequent critical input and discussions on this work and P. Alexander, A. Muncey and A. Lloyd for administrative support. Kymab was funded by investments from the Wellcome Trust, London.

\section{AUTHOR CONTRIBUTIONS}

A. Bradley, G.A.F., E.-C.L. and M.J.O. planned and coordinated the project and wrote the manuscript; Q.L. and W.W. devised transgenic strategies and performed experiments; H.L., D.S., S.O., H.A. and A.C. performed molecular biology and created modified and compound BACs; Q.L., D.S., H.H., J.P., L. Bayliss and A. Beasley performed all the ES cell manipulations and culturing; H.L., R.B., A.C. and T.B.-G. performed molecular biology and genotyped all ES cell lines and mice; S.C. microinjected ES cells into blastocysts and maintained the mouse colonies; D.S. and B.F. analyzed mouse immune cells by FACS; A.W. and H.L. sequenced antibody transcripts of full repertoires; N.E. performed bioinformatic and statistical analysis; S.H. planned $\mathrm{mAb}$ discovery experiments; V.G., I.K., J.C. and A.D. conducted and coordinated all antibody discovery and characterization; A.L., H.A., A.M., N.W., D.S. and L.T. performed functional assays on mAbs; M.K., L. Bonoli, B.F. and R.S. cloned antibody heavy and light chains; Q.L., V.W., S.W. and A.F.-D. created hybridomas for mAb discovery; and A.S. performed peripheral immune cell analysis.

\section{COMPETING FINANCIAL INTERESTS}

The authors declare competing financial interests: details are available in the online version of the paper.
Reprints and permissions information is available online at http://www.nature.com/ reprints/index.html.

1. Beck, A., Wurch, T., Bailly, C. \& Corvaia, N. Strategies and challenges for the nex generation of therapeutic antibodies. Nat. Rev. Immunol. 10, 345-352 (2010).

2. Neuberger, M.S. et al. Diversification and selection mechanisms for the production of protein repertoires: lessons from the immune system. Appl. Biochem. Biotechnol. 83, 53-62 (2000).

3. Lonberg, N. Human antibodies from transgenic animals. Nat. Biotechnol. 23 , 1117-1125 (2005)

4. Lee, E.C. \& Owen, M. The application of transgenic mice for therapeutic antibody discovery. Methods Mol. Biol. 901, 137-148 (2012).

5. Reichert, J.M. Marketed therapeutic antibodies compendium. MAbs 4, 413-415 (2012).

6. Taylor, L.D. et al. A transgenic mouse that expresses a diversity of human sequence heavy and light chain immunoglobulins. Nucleic Acids Res. 20, 6287-6295 (1992).

7. Varriale, S., Merlino, A., Coscia, M.R., Mazzarella, L. \& Oreste, U. An evolutionary conserved motif is responsible for immunoglobulin heavy chain packing in the B cell membrane. Mol. Phylogenet. Evol. 57, 1238-1244 (2010).

8. Lonberg, N. et al. Antigen-specific human antibodies from mice comprising four distinct genetic modifications. Nature 368, 856-859 (1994).

9. Green, L.L. et al. Antigen-specific human monoclonal antibodies from mice engineered with human Ig heavy and light chain YACs. Nat. Genet. 7, 13-21 (1994).

10. Fishwild, D.M. et al. High-avidity human IgG kappa monoclonal antibodies from a novel strain of minilocus transgenic mice. Nat. Biotechnol. 14, 845-851 (1996).

11. Han, C. et al. Comprehensive analysis of reproductive ADAMs: relationship of ADAM4 and ADAM6 with an ADAM complex required for fertilization in mice. Biol. Reprod. 80, 1001-1008 (2009).

12. Osoegawa, K. et al. A bacterial artificial chromosome library for sequencing the complete human genome. Genome Res. 11, 483-496 (2001).

13. Lee, E.C. et al. A highly efficient Escherichia coli-based chromosome engineering system adapted for recombinogenic targeting and subcloning of BAC DNA. Genomics 73, 56-65 (2001).

14. Schlake, T. \& Bode, J. Use of mutated FLP recognition target (FRT) sites for the exchange of expression cassettes at defined chromosomal loci. Biochemistry 33 , 12746-12751 (1994).

15. Yusa, K. et al. Targeted gene correction of alpha1-antitrypsin deficiency in induced pluripotent stem cells. Nature 478, 391-394 (2011).

16. Ramirez-Solis, R., Liu, P. \& Bradley, A. Chromosome engineering in mice. Nature 378, 720-724 (1995).

17. Ekiert, D.C. et al. Cross-neutralization of influenza A viruses mediated by a single antibody loop. Nature 489, 526-532 (2012).

18. Karpusas, M. et al. Structure of CD40 ligand in complex with the Fab fragment of a neutralizing humanized antibody. Structure 9, 321-329 (2001).

19. Rudolf, M. \& Koch, H. Human monoclonal antibody against $S$. aureus derived alpha-toxin and its use in treating and preventing abscess formation. PCT/EP2010/004884, WO/2011/018208 (2011).

20. Tkaczyk, C. et al. Identification of anti-alpha toxin monoclonal antibodies that reduce the severity of Staphylococcus aureus dermonecrosis and exhibit a correlation between affinity and potency. Clin. Vaccine Immunol. 19, 377-385 (2012).

21. Sellman, B.R. et al. Antibodies that specifically bind Staphylococcus aureus alpha toxin and methods of use. PCT/US2012/024201, WO/2012/109285 (2006).

22. Fraser, P. \& Grosveld, F. Locus control regions, chromatin activation and transcription. Curr. Opin. Cell Biol. 10, 361-365 (1998)

23. Tomizuka, K. et al. Functional expression and germline transmission of a human chromosome fragment in chimaeric mice. Nat. Genet. 16, 133-143 (1997).

24. Hernandez, D., Mee, P.J., Martin, J.E., Tybulewicz, V.L. \& Fisher, E.M. Transchromosomal mouse embryonic stem cell lines and chimeric mice that contain freely segregating segments of human chromosome 21. Hum. Mol. Genet. 8, 923933 (1999).

25. Radaev, S. et al. Structural and functional studies of $\lg \alpha \beta$ and its assembly with the B cell antigen receptor. Structure 18, 934-943 (2010).

26. Osborn, M.J. et al. High-affinity IgG antibodies develop naturally in Ig-knockout rats carrying germline human $\mathrm{IgH} / \mathrm{lg} / \mathrm{lg} \lambda$ loci bearing the rat $\mathrm{CH}$ region. J. Immunol. 190, 1481-1490 (2013).

27. Muyrers, J.P., Zhang, Y., Testa, G. \& Stewart, A.F. Rapid modification of bacterial artificial chromosomes by ET-recombination. Nucleic Acids Res. 27, 1555-1557 (1999).

28. Prosser, H.M., Rzadzinska, A.K., Steel, K.P. \& Bradley, A. Mosaic complementation demonstrates a regulatory role for myosin VIla in actin dynamics of stereocilia. Mol. Cell. Biol. 28, 1702-1712 (2008).

29. Featherstone, K., Wood, A.L., Bowen, A.J. \& Corcoran, A.E. The mouse immunoglobulin heavy chain V-D intergenic sequence contains insulators that may regulate ordered V(D)J recombination. J. Biol. Chem. 285, 9327-9338 (2010).

30. Sattentau, Q.J. Vaccinology: a sweet cleft in HIV's armour. Nature 480, 324-325 (2011).

31. Giudicelli, V. et al. IMGT, the international ImMunoGeneTics database. Nucleic Acids Res. 25, 206-211 (1997). 


\section{ONLINE METHODS}

Construction of landing-pad vectors and BACs. The first step in S-RMCE is to target a landing-pad construct to the site of insertion between the most $3^{\prime}$ endogenous $\mathrm{J}_{\mathrm{H}}$ and $\mathrm{J}_{\mathrm{K}}$ segments and the $\mathrm{E} \mu$ or $\mathrm{iE \kappa}$, respectively. The parental landing-pad vector was constructed by inserting a human HPRT minigene between inverted piggyBac terminal repeats (PB5'ITR). The HPRT minigene consists of a $P G K$ promoter, HPRT exons 1 and $2\left(5^{\prime}-H P R T\right)$ flanked by lox5171 and loxP sites, followed by the remaining exons (3-9) of HPRT $\left(3^{\prime}-H P R T\right)$. To target each landing pad to a specific genomic location, we retrieved flanking homology arms from relevant BACs containing mouse genomic DNA. Each homology region was retrieved using recombineering ${ }^{13}$ and then inserted into the relevant parental landing pad vector. For the heavy-chain locus, the homology arms correspond to coordinates Chr. 12: 113,428,225-113,432,739 (5') and Chr. 12: 113,425,850-113,428,224 (3'; all coordinates herein are based on the December 2011 GRCm38/mm 10 assembly). The kappa light-chain homology arms correspond to coordinates Chr. 6 : 70,720,234-70,724,761 (5') and Chr. 6: 70,724,743-70,729,324 (3'). The mouse Igh-containing BAC (bMQ451a16) and mouse Igk-containing BAC (bMQ126m16) were obtained from the Wellcome Trust Sanger Institute BAC clone archives.

S-RMCE was used to insert five human heavy-chain BACs (three standardlength BACs and two composite BACs derived from the fusion of two adjacent BACs), five normal human kappa light-chain BACs and five human lambda light-chain BACs (four standard-length and one composite BAC) into the mouse genome at the site of the landing pads. These insertions and their inverted derivatives devoid of selection markers were transferred into the mouse germline by ES cell technology. The HK mouse and HL mice described here are both homozygous for the heavy-chain locus with three human BACs inserted with the inversion. The HK and HL mice are also homozygous for humanized kappa and light-chain loci, respectively, each with three human BACs inserted; HL also has an inactivation of the kappa locus. In order to insert these BACs in a stepwise process, the ends of each have to be modified for use in the S-RMCE system. Unmodified human BACs were derived from the RPC1-11 library and obtained from Invitrogen (Life Technologies). The $I G H$ BACs included the set of $\mathrm{V}_{\mathrm{H}}$ genes from $\mathrm{V}_{\mathrm{H}} 6-1$ to $\mathrm{V}_{\mathrm{H}} 2-26$, and the IGK BACs included the set of $V_{\kappa}$ genes from $V_{\kappa} 4-1$ to $V_{\kappa} 2-40$. The sizes of the BACs and their respective coordinates within the human genome are detailed in Supplementary Table 6. Each BAC was modified by recombineering at both ends in preparation for S-RMCE, and the overlapping regions were trimmed so that the genomic structure of the final insertion was contiguous, as it would normally be in the human genome. Modifying elements were constructed for the $5^{\prime}$ and $3^{\prime}$ modifications that included features such as loxP (wild-type and mutant) sites, piggyBac transposon ITR elements, a partial HPRT gene and selection markers (puro $\Delta T K$ and $n e o^{\mathrm{R}}$ genes). Homology arms allowing these modifying elements to be targeted into the BACs by recombineering flanked each modifying element (Fig. 1a). The site chosen for insertion of the modifying elements allowed for the trimming of overlapping elements present in adjacent human BACs. In some instances two adjacent BACs did not overlap and would leave a gap of otherwise contiguous human genomic DNA. In these cases, the modifying vectors were constructed to include the missing DNA such that proper S-RMCE (with removal of modifying elements) would leave a contiguous human genomic fragment inserted into the mouse genome.

Once the $5^{\prime}$ and $3^{\prime}$ modifying elements were targeted in a predefined region within a given $\mathrm{BAC}$, the modified $\mathrm{BAC}$ was ready to be electroporated into mouse ES cells. Insertion of the BAC was facilitated by Cre-mediated recombination between like lox sites (wild-type to wild-type, mutant to mutant), providing directional control of the insertion. Cre was expressed from a co-transfected plasmid, pCAGGS-cre ${ }^{32}$, and successful insertion events were isolated by resistance to selection medium-containing drugs such as puromycin, as correct insertion events result in creation of a functional, expressed selection marker (Fig. 1a).

The mouse Igh and $I g k$ loci were inactivated by inversion after the human $\mathrm{V}(\mathrm{D}) \mathrm{J}$ or $\mathrm{VJ}$ segments were inserted by S-RMCE. The third BAC insertion (as with any odd number of BAC insertions) resulted in a landing pad with a loxP site and a $3^{\prime} H P R T$ segment immediately $5^{\prime}$ to the inserted human variable immunoglobulin sequences. This landing pad marked the $3^{\prime}$ end of the inversion, whereas the $5^{\prime}$ end was delimited by a vector containing a loxP element targeted into a distant region of the same chromosome that sequence analysis indicated was devoid of functional genes. A region $6.25 \mathrm{Mbp}$ distant was chosen for the Igh locus and one $26.3 \mathrm{Mbp}$ distant for the Igk locus. In addition to an inverted loxP site, the $5^{\prime}$ inversion endpoint included a $5^{\prime} H P R T$ segment. Cre-mediated recombination between the inverted $\operatorname{lox} \mathrm{P}$ sites in targeted ES cells allowed for the precise inversion of the endogenous mouse immunoglobulin variable region away from the inserted human immunoglobulin variable regions. Correct inversion events are selected by acquisition of a functional HPRT gene, as the $5^{\prime}$ and $3^{\prime}$ segments are brought into functional alignment in proper inversion events.

ES cell engineering. The procedures for ES cell culture, electroporation and drug selection have been described previously ${ }^{33}$. Mouse male AB2.1 (129S7/SvEvBrd-Hprt $\left.t^{\mathrm{b}-\mathrm{m} 2}\right)$ ES cells were used for targeting ${ }^{16}$. ES cells were cultured in M15 medium (knockout DMEM supplemented with 15\% FBS, $2 \mathrm{mM}$ glutamine and $100 \mu \mathrm{M} \beta$-mercaptoethanol) and maintained on irradiated SNL76/7 feeders ${ }^{34}$. All cell lines in this study were tested for mycoplasma contamination and were negative. All electroporation was conducted using a Bio-Rad electroporator (GenePulser Xcell) at $500 \mu \mathrm{F}$ and $230 \mathrm{~V}$.

S-RMCE consists of three steps: targeting of the landing pad, insertion of BACs and excision of $3^{\prime}$ modified cassette. To target the landing pad into the mouse genome, ES cells $\left(0.9 \times 10^{7}\right.$ cells $)$ were electroporated with linearized landing-pad vector DNA. Immediately before electroporation, cell suspensions of $0.9 \times 10^{7}$ cells and $20 \mu \mathrm{g}$ of linearized targeting-vector DNA were mixed in a final volume of $900 \mu \mathrm{l}$ PBS. After electroporation, cells were seeded onto three $10-\mathrm{cm}$ plates (with feeders), and colonies were picked after $7 \mathrm{~d}$ of selection in HAT ( $100 \mu \mathrm{M}$ sodium hypoxanthine, $0.4 \mu \mathrm{M}$ aminopterin and $16 \mu \mathrm{M}$ thymidine) and $3 \mathrm{~d}$ in $\mathrm{HT}(100 \mu \mathrm{M}$ sodium hypoxanthine and $16 \mu \mathrm{M}$ thymidine). Colonies were picked into 96-well plates for expansion and genotyping by long-range PCR.

For the insertion of BACs by S-RMCE, $0.9 \times 10^{7}$ cells targeted with the landing pad were electroporated with $20 \mu \mathrm{g}$ modified BAC DNA and $10 \mu \mathrm{g}$ pCAGGS-Cre ${ }^{32}$. The selection of ES cell clones with puromycin $\left(2 \mu \mathrm{g} \mathrm{ml}^{-1}\right)$ began $72 \mathrm{~h}$ after transfection and continued for 9-11 d. Colonies were picked into 96-well plates for expansion and genotyping. Correct BAC integration was confirmed by PCR strategies that identified correct junction fragments. The integrity of inserted BACs was confirmed by PCR with a series of locusspecific primers covering the relevant human $V(D) J$ genes.

The $3^{\prime}$ modification was removed from the ES cell genome by piggyBac transposase-induced transposition ${ }^{15}$. ES cells from one well of a 24 -well plate were electroporated with $5 \mu \mathrm{g}$ of piggyBac transposase plasmid and seeded onto one 24-well plate with feeders. The plates were maintained under normal ES cell conditions for $3 \mathrm{~d}$ before the cells were trypsinized into single cells and seeded onto $10-\mathrm{cm}$ plates at low density (100-500 cells per plate). FIAU selection was initiated $24 \mathrm{~h}$ after low-density plating and maintained for 10-12 d. Excision of the selection marker was confirmed by PCR of the genomic DNA junction formed after excision.

The inversion of the endogenous mouse immunoglobulin variable region was performed after the S-RMCE integration of odd numbers of BACs (Fig. 1b). This process involves three steps-inversion endpoint vector targeting, Cremediated inversion, and finally excision of both modified cassettes at the inversion endpoints. As described for landing-pad targeting, ES cells were electroporated with linearized endpoint targeting vectors for either the mouse $I g h$ or $I g k$ loci as appropriate. ES cells $\left(0.9 \times 10^{7}\right)$ and $20 \mu \mathrm{g}$ of linearized vector DNA were used per transfection. The selection of ES cell clones with puromycin $\left(3 \mu \mathrm{g} \mathrm{ml}^{-1}\right)$ began $24 \mathrm{~h}$ after transfection and proceeded for 11-13 d. Colonies were picked into 96-well plates for expansion and genotyping by longrange PCR. Clones with correctly targeted $5^{\prime}$ inversion endpoints were expanded for the Cre-mediated inversion of endogenous mouse genes. $0.9 \times 10^{7} \mathrm{ES}$ cells were electroporated with $10 \mu \mathrm{g}$ pCAGGS-Cre and seeded onto three $10-\mathrm{cm}$ plates (with feeders), and colonies were picked after $7 \mathrm{~d}$ of selection in $100 \mu \mathrm{M}$ sodium hypoxanthine, $0.4 \mu \mathrm{M}$ aminopterin and $16 \mu \mathrm{M}$ thymidine (HAT) and $3 \mathrm{~d}$ in $100 \mu \mathrm{M}$ sodium hypoxanthine and $16 \mu \mathrm{M}$ thymidine (HT). Inversion of the endogenous genes were confirmed by PCR of the new genomic DNA junctions expected after inversion. The cassettes containing the HPRT gene and the puromycin and neomycin selection markers were removed from the genome by piggyBac transposase-induced transposition (Fig. 1b). ES cells 
in one well of a six-well plate were electroporated with $10 \mu \mathrm{g}$ of piggyBac transposase plasmids and seeded onto one six-well plate with feeders. The plates were maintained under normal ES cell conditions for $3 \mathrm{~d}$ before the cells were trypsinized into single cells and seeded onto $10-\mathrm{cm}$ plates at low density (100-500 cells per plate). FIAU selection was initiated $24 \mathrm{~h}$ later and maintained for 10-12 d. Excision of the selection marker was confirmed by PCR of junctions formed after excision of the HPRT minigene and puromycin or neomycin selection markers (Fig. 1). PCR was also used to confirm that the excision of the piggyBac elements was not followed by reinsertion of the element elsewhere in the genome.

Mouse generation. Positive ES cell clones were injected into blastocysts from the C57BL/6 Tyr $r^{c-B r d}$ mouse strain by standard procedures ${ }^{33}$. Injected blastocysts were transferred to the uteri of pseudopregnant female B6/CBA $\mathrm{F}_{1}$ recipients. Approximately 40 blastocysts were injected for each clone. Highpercentage chimeras were mated with albino C57BL/6 $\mathrm{Tyr}^{\mathrm{c}-\mathrm{Brd}}$ females to allow identification of pups derived from the injected ES cells. Offspring were of mixed 129S7 and C57BL/6J genetic backgrounds. Transgenic mice were identified by PCR of genomic DNA from ear-clip biopsies designed to detect the junction resulting from the excision of the $3^{\prime}$ modification after the first BAC insertion-in other words, the junction of the $3^{\prime}$ end of inserted human DNA and the adjacent mouse genome. The integrity of the inserted BACs in resulting transgenic animals was confirmed by PCR with a series of locusspecific primers covering all the relevant human $\mathrm{V}(\mathrm{D}) \mathrm{J}$ genes. $\mathrm{F}_{1}$ mice with humanized heavy- or light-chain alleles were intercrossed once or twice to generate double (heavy-chain and kappa light-chain insertion alleles in HK mice) or triple (heavy-chain and lambda light-chain insertion alleles and the kappa null allele in HL mice) heterozygotes. Selected mice were interbred for one or more generations to produce mice that were homozygous for all the engineered heavy- and light-chain loci.

All animal experimentation and husbandry was conducted under approval of the Wellcome Trust Sanger Institute AWERB (Animal Welfare and Ethics Review Body). United Kingdom Home Office approval is provided under Project License 80/2432.

Deep sequencing analysis of antibody-coding transcripts. RNA was isolated using Trizol (Invitrogen) from mouse spleen or from human peripheral blood mononuclear cells enriched for naive B cells using the Human Naive B cell Isolation Kit II (Miltenyi). $5^{\prime}$ RACE was performed on $5 \mu \mathrm{g}$ of total RNA to generate CDNA with a known sequence at the $5^{\prime}$ end using the ExactSTART Eukaryotic mRNA 5' - \& 3' RACE kit (Epicentre) using the manufacturer's protocol with the following modifications. The $5^{\prime}$ RACE acceptor oligo was replaced with an RNA oligo (P7 RNA oligo: AGACGUGUGCUCUUCCGAUCU) specific to a 21-bp portion of the Illumina P7 adaptor. The first-strand cDNA was synthesized using reverse primers specific for the first exon of the constant region. The primers include, for heavy-chain transcripts in transgenic animals, an $\operatorname{lghm}$ reverse transcription (RT) primer (GAAGACATTTGGGAAGGACTG) and an lghg1 RT primer (GGCCAGTGGATAGACAGATG), and for the light chains in transgenic animals, an equimolar mix of an $l g k$ RT primer (TGGGAAGATGGATACAGTTGG), a mouse $I g l$ RT primer (CCTTCCAGKCCACTGTCAC) and a human IGL RT primer (CTCCCGGG TAGAAGTCACT). The primers include, for human samples, a human IGM RT primer (ACGGGGAATTCTCACAGG) and a human IGK RT primer (GGGAAGATGAAGACAGATGGT). Second-strand synthesis and enrichment of $\mathrm{V}(\mathrm{D}) \mathrm{J}$ sequences was achieved by performing 8 cycles of PCR using a primer specific to the ligated RNA oligo (P7 PCR 1: [GTGACTGGAGTTC]AGACGTG TGCTCTTCCGATCT) that included an additional Illumina P7 sequence at the 5 ' end (delimited by square brackets). This primer was paired with one specific to the constant region-J segment splice junction appropriate to the RT primer used previously. (IgM P5 PCR 1: GGGAAGGACTGACTCTCTGA; IgG1 P5 PCR 1: GGTGTCGTTTTGGCTGA; Igא P5 PCR 1: CAGCATCAGCCCGTTT; human Ig $\lambda$ 1-6 P5 PCR 1: GGCGGGAACAGAGTGAC; human Ig $\lambda 7$ P5 PCR 1: AGGGTGGGAACAGAGTGAC; mouse Ig $\lambda$ P5 PCR 1: GAAGGT GGAAACAVGGTGA; human IgM P5 PCR1: CGGATGCACTCCCTGA; human Igא P5 PCR1: GCAGCCACAGTTCGTTT). All the P5 PCR primers listed had the following additional Illumina P5 sequence, including two bases of random nucleotides at the $3^{\prime}$ end: ACACTCTTTCCCTACACGACGCTC TTCCGATCTNN.

Products from these PCR reactions were purified using AMPure XP beads (Beckman Coulter) according to the manufacturer's protocol. A second round of 8-15 cycles of PCR was performed to add the remaining portions of the Illumina P5 \& P7 adaptor sequences and to incorporate a barcode to enable sample multiplexing. The forward primer (P5 PCR 2: [AATGATACGGCGA CCACCGAGATCT]ACACTCTTTCCCTACACGACGCTCTT) was specific to the P5 sequence with the additional P5 flow-cell binding sequence shown within square brackets. The reverse primer (P7 PCR 2 index X: [CAAGCAG AAGACGGCATACGAGAT*****] GTGACTGGAGTTCAGACGTGT) was specific to the P7 sequence and incorporated a 6-bp barcode (denoted by asterisks) and the P7 flow-cell binding sequence shown within square brackets. The 6-bp barcodes were standard Illumina Truseq 6-bp indexes. The final PCR product was purified using AMPure XP beads (Beckman Coulter) and consisted of a library of $\mathrm{V}(\mathrm{D}) \mathrm{J}$ transcribed genes with a complete P5 and P7 Illumina adapters on either end. The V(D)J libraries were sequenced using a $2 \times 250$-bp paired-end run on an Illumina Mi-Seq machine.

Raw sequence data were converted into fastq from BAM files using bam2 fastq. The sequences were then binned into separate files (for IgM, IgG1, IgK and Ig $\lambda$ ) by constant region using the Kymab seq-utils program. Seq-utils is a Java program that automates the constant-region binning and identifies the $\mathrm{V}(\mathrm{D}) \mathrm{J}$ segments in large sets of antibody heavy-chain transcript sequences. The program uses BLASTN to assign the best matching $\mathrm{V}$ and $\mathrm{J}$ segments and then uses BLASTN configured for short sequences to find a contiguous, nongapped alignment between the transcript and the closest germline D segment, looking only at the nucleotides between the matched $\mathrm{V}$ and J segments. The CDR1 and CDR2 regions are identified by alignment to the reference sequence. The CDR3 is identified based on the conserved TATTACTGT and CTGGGG motifs, and the P- and N-nucleotide insertions and any deletions are then determined for the two junctions.

For each analysis conducted on these sequence sets, at least 260 individual sequences were included, and in the case of deep sequencing, at least 6,700 sequences. The exact sample size $(n)$ for each data point is provided in the figures. In cases where there is more than one experimental source for sequenced transcripts, the range of experimental results is presented.

Cell staining and flow cytometry analysis. To evaluate the phenotype of HK and HL mice, we compared them to age-matched wild-type mice having a similar combined, randomized 129S7 and C57BL/6 genetic background.

Bone marrow, spleen tissue and lymph nodes were isolated from adult male and female mice (7-18 weeks of age) using aseptic procedures. For each genotype, between 3 and 5 animals were analyzed to account for the possibility of individual anomalous measurements due to the known variability of the immune system. No animals were excluded according to pre-established criteria.

Single-cell suspensions were prepared from the spleen, lymph nodes and bone marrow. Each sample was collected in labeled tubes, and the worker performing the analysis was not blinded to the mouse identification or genotype. For identification of kappa and lambda light chain-expressing cells, cell preparations were stained with allophycocyanin (APC)-conjugated antiCD45R (eBioscience, RA3-6B2), phycoerythrin (PE)-conjugated anti-lambda (Southern Biotech, JC5-1) and fluorescein (FITC)-conjugated anti-kappa (BD Pharmingen, 187.1) antibodies. The mature spleen population was defined using APC-conjugated anti-CD45R, PE-conjugated anti-IgD (eBioscience, 11-26c) and FITC-conjugated anti-IgM (eBioscience, II/41) antibodies. Spleen germinal centers were stained with APC-conjugated anti-CD45R, PE-conjugated anti-CD38 (eBioscience, 90) and FITC-conjugated anti-CD95 (BD Bioscience) antibodies. Spleen marginal zone and follicular B cells were stained using FITC-conjugated anti-CD21/CD35 (BD Pharmingen, 7G6) and PE-conjugated anti-CD23 (BD Pharmingen, B3B4) antibodies. Bone marrow progenitors were defined using APC-conjugated anti-CD45R, FITC-conjugated anti-CD43 (eBioscience, eBioR2/60) and PE-Cy7-conjugated anti-IgM (eBioscience, II/41) antibodies. All staining reactions were incubated at $4{ }^{\circ} \mathrm{C}$ for $20 \mathrm{~min}$ using $1 \times 10^{6}$ cells in $100 \mu \mathrm{l}$ of PBS with $3 \%$ FBS. Samples were washed twice in $400 \mu \mathrm{l}$ PBS with 3\% FBS and analyzed on MACSQuant analyzer (Miltenyi 
Biotec). All acquired data were analyzed using Kaluza software (Beckman Coulter USA).

For peripheral blood analysis, blood was collected from the retro-orbital sinus of anesthetized animals into EDTA-coated pediatric tubes (Kabe Labortechnik GmbH, Numbrecht, Germany). Total white blood cell counts were obtained using a hematology analyzer (Scil Vetabc, Montpellier, France). Whole blood $(25 \mu \mathrm{l})$ was stained with two multicolor antibody cocktails titrated to result in saturating binding for $20 \mathrm{~min}$ at room temperature (antibodies listed in Supplementary Table 7). Samples were then fixed (BD Cell Fix, BD Biosciences, Oxford, UK) and red blood cells lysed before resuspension in FACS buffer and acquisition on a BD LSR II (BD Biosciences). Compensation was calculated using compbeads (UltraComp eBeads, eBioscience, Hatfield, UK) and BD FACSDiva software. Doublets were excluded using FSC-H versus FSC-A gate, and a minimum of 50,000 CD45 ${ }^{+}$singlet leukocyte events were recorded. Samples were analyzed using FlowJo (Tree Star Inc, Ashland, OR, USA) and leukocyte subsets determined as the percentage of singlet $\mathrm{CD} 45^{+}$leukocytes or other cell types (CD4 ${ }^{+} \mathrm{T}$ cells, $\mathrm{CD} 8^{+} \mathrm{T}$ cells, NK cells or monocytes).

Antigens and benchmark antibodies. Recombinant human CD40L was obtained from Peprotech, recombinant Staphylococcus aureus hemolysin alpha (Hla) mutant $\mathrm{H} 35 \mathrm{~L}^{35}$ was purified from E. coli cultures engineered to express the mutant, and native S. aureus Hla was obtained from Sigma (C9903). Ovalbumin (OVA) was also obtained from Sigma (A7641). Benchmark antibodies were constructed based on published sequences, cloned into expression vectors carrying murine IgG1 constant regions and expressed in suspension Chinese hamster ovary $(\mathrm{CHO})$ cell cultures. Murine isotype control antibodies were from Sigma (M9269).

Immunizations. Protein antigens were prepared in phosphate-buffered saline (PBS) at about $1 \mathrm{mg} / \mathrm{ml}$, and the volume corresponding to the desired amount of protein was increased to an injectable volume with PBS and a final concentration of $1 \%$ Alhydrogel (vac-alu-50, Invivogen) and $10 \mu \mathrm{g} \mathrm{CpG} \mathrm{(ODN1826,}$ tlrl-1826, Invivogen). This volume was then mixed 1:1 (v/v) with either Freund's or Sigma as an additional adjuvant (Sigma Adjuvant S6322; Sigma CFA F5881, Sigma IFA F5506; prepared according to manufacturer's instructions). For viscous adjuvants such as Complete Freund's (CFA), the solution was mixed by vortexing or repeated passage through a syringe until a smooth emulsion was formed. Injections were performed using a 1-ml syringe and a 27 -gauge needle in 7 - to 18 -week-old male and female mice. Prime and boost injections were intraperitoneal (i.p.) or subcutaneous (s.c.), depending on the experimental requirements. OVA and CD40L (KM19) immunizations used CFA via s.c. injection for the prime immunization and intermediate boosts used Incomplete Freund's Adjuvant (IFA) via i.p. injection. Hemolysin immunization used Sigma adjuvant via i.p. injection, and the KM31 CD40L immunization used Sigma adjuvant and was injected every second day over 2 weeks via multiple s.c. injections. Volumes varied depending on the injection route and experimental requirements and were in accordance with the relevant UK Home Office animal license for the procedure. Final boosts were delivered without adjuvant intravenously (for i.p. regimes) or s.c. (for s.c. regimes).

Hybridoma generation. Spleens from immunized mice were harvested after mice were killed, homogenized to single-cell suspensions and fused with myeloma SP0/2 cells using a BTX electrofuser (ECM2001). Fused hybridoma cells were cultured overnight and then plated in culture dishes. After about $10 \mathrm{~d}$ of culture, visible colonies were picked and transferred to 96-well culture plates and cultured for a further $3 \mathrm{~d}$ before transfer to 384 -well plates for the primary screen of supernatants.

Serum titer determination. Serum bleeds taken about 1 week after antigen boost were analyzed by a reverse-format binding enzyme-linked immunosorbent assay (ELISA). 96-well immunoassay plates (96w EIA/RIA Costar Corning) were coated with $50 \mu \mathrm{l} /$ well anti-mouse IgG antibody (Southern Biotech 1030-01) at $4 \mu \mathrm{g} / \mathrm{ml}$ in PBS overnight at $4{ }^{\circ} \mathrm{C}$. Plates were washed 3 times with PBS/Tween $(0.1 \% \mathrm{v} / \mathrm{v})$ and blocked with PBS/BSA ( $1 \% \mathrm{w} / \mathrm{v})$ for $1 \mathrm{~h}$ at room temperature. After being washed again as above, $50 \mu \mathrm{l}$ of serially diluted serum samples in PBS/BSA $(0.1 \% \mathrm{w} / \mathrm{v})$ were added to wells and incubated for $1 \mathrm{~h}$ at room temperature. After incubation, plates were again washed as above and incubated with $50 \mu \mathrm{l}$ of biotinylated antigen at $0.1 \mu \mathrm{g} / \mathrm{ml}$ in PBS/BSA $(0.1 \% \mathrm{w} / \mathrm{v})$ for $1 \mathrm{~h}$ at room temperature. Plates were washed again and $50 \mu \mathrm{l}$ of streptavidin-HRP (Sigma S5512) added at 1/50,000 dilution in $\mathrm{PBS} / \mathrm{BSA}(0.1 \% \mathrm{w} / \mathrm{v})$ for $1 \mathrm{~h}$ at room temperature. Plates were washed again as above and developed using $100 \mu \mathrm{l}$ of TMB (Sigma T0440) and stopped using $50 \mu \mathrm{l}$ of $1 \mathrm{M} \mathrm{H}_{2} \mathrm{SO}_{4}$. Absorbance was read at $450 \mathrm{~nm}$.

Surface plasmon resonance. Anti-mouse IgG (GE Healthcare/Biacore) was coupled to the GLM chip (Bio-Rad) by primary amine coupling. The GLM chip was activated using NHS/EDAC and the anti-mouse IgG coupled to this activated surface and then blocked using $1 \mathrm{M}$ ethanolamine. Immobilization was carried out in either HBS-EP (Teknova) or HBS-N (GE Healthcare/Biacore) at room temperature or $37^{\circ} \mathrm{C}$, respectively. The anti-mouse IgG surface on the GLM chip was used to directly capture antibodies of interest. For kinetic analysis, five concentrations of analyte were used $(256 \mathrm{nM}, 64 \mathrm{nM}, 16 \mathrm{nM}$, $4 \mathrm{nM}$ and $1 \mathrm{nM}$ ). For data analysis, the binding sensorgrams were referenced using the internal "interspot" referencing unique to the ProteOn XPR36, which are double referenced using the buffer injection sensorgram. Finally the data were analyzed using the 1:1 model inherent to the ProteOn XPR36 analysis software.

Hybridoma screening. Hybridoma clones producing CD40L-specific antibodies were identified by fluorescent screening. Each well of clear 384-well flat-bottom plates was seeded with $1 \times 10^{4}$ adherent $\mathrm{CHO}$ cells stably transfected with human CD40L in $80 \mu \mathrm{l}$ of F12 medium containing $10 \%(\mathrm{v} / \mathrm{v})$ FBS $\left(1.25 \times 10^{5} \mathrm{cells} / \mathrm{ml}\right)$ using a Multidrop instrument. Cells were incubated overnight at $37^{\circ} \mathrm{C}$ in a $\mathrm{CO}_{2}$ incubator. The next day the culture medium was removed by aspiration and $45 \mu \mathrm{l}$ of LI-COR IRDye $800 \mathrm{CW}$ anti-mouse antibody added at $500 \mathrm{ng} / \mathrm{ml}+5 \mathrm{mM}$ DRAQ5 (LI-COR) diluted 1:25,000 in FACS buffer (PBS $\left.+1 \% \mathrm{BSA}+0.1 \% \mathrm{NaN}_{3}\right) .5 \mu$ of hybridoma supernatant, $5 \mu \mathrm{l}$ of control antibody $(2 \mu \mathrm{g} / \mathrm{ml})$ in hybridoma medium or $5 \mu \mathrm{l}$ of mouse IgG1 control antibody (Sigma M9269, $2 \mu \mathrm{g} / \mathrm{ml}$ ) in hybridoma medium was added using a FluidX liquid handler. Plates were incubated for $1 \mathrm{~h}$ at $4{ }^{\circ} \mathrm{C}$ and culture medium aspirated. The reaction was stopped and the cells fixed by the addition of $25 \mu \mathrm{l}$ of $4 \%$ paraformaldehyde per well and incubation for $15 \mathrm{~min}$ at room temperature. Plates were washed twice with $100 \mu \mathrm{l}$ of PBS, and the wash solution was removed by blotting on paper towels. Plates were scanned using a Li-Cor Odyssey Classic instrument.

Homogeneous time-resolved fluorescence (HTRF) assays were used to test hybridoma supernatants for the presence of anti-Hla and OVA antibodies. $5 \mu \mathrm{l}$ of hybridoma supernatant was transferred to 384 -well assay plates. Each plate included a positive-control antibody $(10 \mu \mathrm{g} / \mathrm{ml}$ diluted in hybridoma medium) and hybridoma medium alone as a negative control. Using a Beckman BioRAPTR each well of the assay plate received $10 \mu \mathrm{l}$ of biotinylated OVA or Hla for a final concentration of $40 \mathrm{ng} /$ well. Plates were incubated at room temperature for $1 \mathrm{~h}$. For detection, $5 \mu \mathrm{l}$ of Europium Cryptate and $5 \mu \mathrm{l}$ of D2 acceptor were added to each well using the Beckman BioRAPTR. Plates were incubated for at room temperature for $1 \mathrm{~h}$ and read on a BMG Pherastar FS reader.

CD40L reporter gene assay. HEK-Blue CD40 cells (Invivogen) were prepared at $2.5 \times 10^{5} \mathrm{cells} / \mathrm{ml}$ in test medium (DMEM $+10 \%$ heat-inactivated FBS + PenStrep $+100 \mu \mathrm{g} / \mathrm{ml}$ Normocin). $40 \mu$ of this cell suspension was distributed to each well of a 384-well plate for a final density of $1 \times 10^{4} \mathrm{cells} /$ well. To each of these wells, $5 \mu \mathrm{l}$ of recombinant CD40L (R\&D) at $50 \mathrm{ng} / \mathrm{ml}$ were added. In quadrant 1 and $2,5 \mu \mathrm{l}$ of hybridoma supernatant was added to each well. For controls, $5 \mu \mathrm{l}$ of positive benchmark mAb or murine IgG1 isotype control (Sigma M9269) was added, both at a final concentration of $200 \mathrm{ng} / \mathrm{ml}$ in hybridoma medium. The benchmark antibody is $5 c 8$, which is a humanized IgG1 antibody that went into clinical development as ruplizumab (Biogen Idec, Cambridge, $\mathrm{MA}$ ). The published humanized $\mathrm{V}_{\mathrm{H}}$ and $\mathrm{V}_{\mathrm{K}}$ regions of $5 \mathrm{C} 8$ (ref. 18) were synthesized and cloned with murine IgG1 and kappa constant regions, respectively, and recombinantly expressed in $\mathrm{CHO}$ cells to match the chimeric format of antibodies generated by the transgenic mice. In quadrant 3 and 4, $5 \mu \mathrm{l}$ of hybridoma supernatant was added diluted 1:2 in PBS. Plates were incubated at $37^{\circ} \mathrm{C}$ in a $\mathrm{CO}_{2}$ incubator for $18-24 \mathrm{~h}$. QUANTI-Blue solution (Invivogen) was prepared according to the manufacturer's instructions, 
and $40 \mu \mathrm{l}$ added per well to a white flat-bottom 384-well plate. $10 \mu \mathrm{l}$ of the contents of each assay and control well was transferred to the corresponding well containing QUANTI-Blue solution and incubated in the dark at room temperature for $1.5 \mathrm{~h}$. Resulting secreted alkaline phosphatase (SEAP) levels were determined using a Biotek Synergy spectrophotometer at $640 \mathrm{~nm}$. Each hybridoma supernatant and control antibody was tested in duplicate. $\mathrm{IC}_{50}$ values were calculated from a nonlinear four-parameter curve fit using GraphPad Prism software.

Rabbit red blood cell lysis assay. Recombinant antibodies at $7.5 \mu \mathrm{g} / \mathrm{ml}$ in PBS were pipetted into wells of a 96-well plate and serially diluted 1:2 in PBS (100 $\mu \mathrm{l}$ total). Each dilution was mixed with $20 \mu \mathrm{l}$ of hemolysin (Sigma C9903; final concentration $0.1 \mu \mathrm{g} / \mathrm{ml}$ ). A negative control (PBS only) and a positive control (PBS + hemolysin) were also included. The benchmark mAb, KBSA-301, is a derivative of a human IgG1 antibody discovered by Kenta Biotech (Zurich, Switzerland) and now under clinical development as AR-301 by Aridis Pharmaceuticals (San Jose, $C A$ ). The $V_{H}$ and $V_{K}$ region sequences were derived from published information ${ }^{19}$, synthesized and cloned with murine IgG1 and kappa constant regions, respectively, and recombinantly expressed in $\mathrm{CHO}$ cells to match the chimeric format of antibodies generated by the transgenic animals. $50 \mu \mathrm{l}$ of $5 \%$ rabbit red blood cells (Tissue Solutions, BLHNZI-505-D) were added to wells of a 96 -well round-bottom plate and $50 \mu \mathrm{l}$ of the sample mixtures and controls added to the cells in duplicate and incubated $60 \mathrm{~min}$ at $37^{\circ} \mathrm{C}$. Plates were then centrifuged $5 \mathrm{~min}$ at 5,000 r.p.m. and $50 \mu \mathrm{l}$ of supernatant transferred into a new 96 -well flat-bottom plate. Absorbance was read at $580 \mathrm{~nm}$. Each recombinant antibody and control antibodies were tested in duplicate, and the experiment was repeated twice on different days. $\mathrm{EC}_{50}$ values were calculated from data from both experiments by a nonlinear fourparameter curve fit using GraphPad Prism software.

32. Araki, K., Imaizumi, T., Okuyama, K., Oike, Y. \& Yamamura, K. Efficiency of recombination by Cre transient expression in embryonic stem cells: comparison of various promoters. J. Biochem. 122, 977-982 (1997).

33. Ramirez-Solis, R., Davis, A.C. \& Bradley, A. Gene targeting in embryonic stem cells Methods Enzymol. 225, 855-878 (1993).

34. McMahon, A.P. \& Bradley, A. The Wnt-1 (int-1) proto-oncogene is required for development of a large region of the mouse brain. Cell 62, 1073-1085 (1990).

35. Menzies, B.E. \& Kernodle, D.S. Site-directed mutagenesis of the alpha-toxin gene of Staphylococcus aureus: role of histidines in toxin activity in vitro and in a murine model. Infect. Immun. 62, 1843-1847 (1994). 